\title{
Desigualdades socioeconômicas e espaciais nas condições de acesso à internet por adolescentes de escolas estaduais
}

Socioeconomic and space inequalities in conditions of access to the internet by adolescents from

state schools

Desigualdades socioeconómicas y espaciales en las condiciones de acceso a internet por adolescentes de las escuelas estatales

\author{
Ângela Luiza Cunha Legey \\ ORCID: https://orcid.org/0000-0002-4295-4182 \\ Universidade Federal do Paraná, Brasil \\ E-mail: angelalegey@gmail.com \\ Aichely Rodrigues da Silva \\ ORCID: https://orcid.org/0000-0001-9447-2380 \\ Universidade Federal do Maranhão, Brasil \\ E-mail: aichely@hotmail.com \\ Aline Daniela da Cruz e Silva \\ ORCID: https://orcid.org/0000-0003-1159-5196 \\ Faculdade Tecnológica do Paraná, Brasil \\ E-mail: alinedaniela.w@gmail.com \\ Christiane Opuszka Machado \\ ORCID: https://orcid.org/0000-0002-1676-4342 \\ Secretaria Municipal de Saúde Curitiba, Brasil \\ E-mail: opuschri@uol.com.br \\ Sarina Giongo Antoniassi \\ ORCID: https://orcid.org/0000-0002-4632-8840 \\ Universidade Federal do Paraná, Brasil \\ E-mail: sarina.giongo@yahoo.com.br \\ Doroteia Aparecida Höfelmann \\ ORCID: https://orcid.org/0000-0003-1046-3319 \\ Universidade Federal do Paraná, Brasil \\ E-mail: doroteia.hofelmann@ufpr.br
}

\section{Resumo}

Objetivou-se analisar condições de acesso à internet por adolescentes de escolas estaduais de Curitiba (PR), e identificar desigualdades de acordo com características demográficas dos alunos e renda do entorno escolar. Associações entre condições de acesso e renda investigadas por 1) modelos de regressão logística multinível para estimar as razões de chance (OR) e intervalos de confiança de 95\% (IC95\%); e 2) índices de Moran Global e local. Nas escolas 1.232 alunos preencheram o questionário. $\mathrm{O}$ dispositivo de acesso à internet mais frequente foi o celular $(75,4 \%)$, seguido do computador $(27,3 \%)$ e tablet $(8,4 \%) ; 81,0 \%$ referiram acessar à internet no domicílio, $25,1 \%$ redes wi-fi; $77,2 \%$ relataram acesso diário, e 53,1\% dispositivo próprio. As chances de acessar à internet pelo celular (OR 1,94 IC95\%1,25; 3,01), diariamente (OR 3,37; IC95\% 1,38; 8,20), e com dispositivos próprios (OR 1,81 IC95\%1,00-3,27) aumentaram com a renda do entorno escolar, enquanto o uso do computador foi menor nos tercis de maior renda (OR 0,61 IC95\% 0,41; 0,91). Observou-se correlação espacial inversa entre renda e uso do celular $(-0,26)$ e tablet $(-0,29)$, e positiva para computador $(0,31)$ e uso diário da internet $(0,30)$. Destaca-se desigualdades nas condições de acesso à internet com características dos alunos e renda.

Palavras-chave: Internet; Fatores socioeconômicos; Desigualdades; Adolescentes.

\section{Abstract}

The objective is to analyze conditions of access to the Internet by adolescents from state schools in Curitiba (PR), and to identify inequalities according to the demographic characteristics of students and income from school neighborhood. Associations between access conditions and income were investigated by: 1) multilevel logistic regression models to estimate odds ratios (OR) and 95\% confidence intervals (95\% CI); and 2) Moran Global and local indexes. In schools, 1,232 students completed the questionnaire. The most frequent Internet access device was the cell phone $(75.4 \%)$, followed by the computer $(27.3 \%)$ and tablet $(8.4 \%) ; 81.0 \%$ reported accessing internet at home, $25.1 \%$ wi-fi networks; $77.2 \%$ are related to daily access and $53.1 \%$ have their own device. The odds of 
accessing the Internet by cell phone (OR $1.9495 \%$ CI 1.25; 3.01), daily (OR 3.37; 95\% CI 1.38; 8.20) and with own devices (OR 1, 81 95\% CI 1.00-3.27) increased with increase of school neighborhood income, while computer use was lower in higher income tertiles (OR $0.6195 \%$ CI $0.41 ; 0.91)$. There was an inverse spatial correlation between income and use of cell phone (-0.26) and tablet (-0.29), and positive for computer $(0.31)$ and daily internet use $(0.30)$. We found inequalities in the conditions of access to the Internet with students and income characteristics.

Keywords: Internet; Socioeconomic factors; Inequalities; Adolescents.

\section{Resumen}

El objetivo es analizar las condiciones de acceso a Internet de los adolescentes de las escuelas públicas de Curitiba (PR) e identificar las desigualdades de acuerdo con las características demográficas de los estudiantes y los ingresos de la escuela secundaria. Asociaciones entre condiciones de acceso e ingresos investigadas por 1) modelos de regresión logística multinivel para estimar como odds ratios (OR) e intervalos de confianza del 95\% (IC 95\%); y 2) los índices Moran Global y local. En las escuelas, 1.232 estudiantes respondieron el cuestionario. El dispositivo de acceso a Internet más frecuente fue el teléfono celular (75.4\%), seguido por la computadora (27.3\%) y la tableta (8.4\%); El 81.0\% informó haber accedido a Internet en el hogar, el $25.1 \%$ a redes wifi; El $77.2 \%$ está relacionado con el acceso diario y el $53.1 \%$ tiene su propio dispositivo. Como las posibilidades de acceder a Internet por teléfono celular (OR 1.94 CI 95\% 1.25; 3.01), diariamente (OR 3.37; CI 95\% 1.38; 8.20) y con dispositivos únicos ( OR 1, 81 IC 95\% 1.00-3.27) aumentó con el ingreso de la escuela secundaria, mientras que el uso de la computadora fue menor en tercios de ingresos más altos (OR 0.61 IC 95\% 0.41; 0.91). Hubo una correlación espacial inversa entre el ingreso y el uso del teléfono celular $(-0.26)$ y la tableta $(-0.29)$, y positivo para la computadora (0.31) y el uso diario de Internet (0.30). Se destacan las desigualdades en las condiciones de acceso a Internet con recursos e ingresos estudiantiles.

Palabras clave: Internet; Factores socioeconómicos; Desigualdades; Adolescentes.

\section{Introdução}

A ampliação do uso de tecnologias digitais nas últimas décadas, vêm promovendo mudanças nas relações sociais, incluindo aquelas relacionadas ao ambiente de trabalho e de estudo. No contexto escolar, as tecnologias da informação representam ferramentas de mediação da aprendizagem, que flexibilizam o processo e promovem a utilização do material conforme as possibilidades de tempo do aluno (Klein et al., 2019).

Apesar da grande evolução tecnológica nos últimos anos, existe expressivo número de indivíduos que não têm acesso à internet. Adicionalmente, são observadas diferenças na qualidade do acesso (Souza \& Dumont, 2018) e na habilidade de utilização das ferramentas de tecnologia da informação (Silva et al., 2019), de acordo com as condições de residência e demográficas (Silva et al., 2019) o que pode refletir no agravamento das desigualdades sociais (Salata et al., 2013).

Informações da Pesquisa Nacional por Amostra de Domicílios Contínua (PNAD) de 2017, indicaram que 74,9\% dos domicílios brasileiros tinham acesso à internet banda larga (Brasil, 2017b). Dos domić́lios entrevistados, 98,7\% utilizavam o celular para acessar à internet, 52,3\% usavam computadores, sendo que 43,3\% utilizavam somente o celular. Os dados ainda demonstram que o número de domicílios com acesso à internet foi crescente em todas as regiões do Brasil, porém de forma desigual, com concentração na região Sudeste. Em 2009, dentre as capitais, Curitiba concentrava a terceira maior quantidade de acessos à internet (4,5\% do total nacional), atrás apenas de São Paulo (16,0\%) e Rio de Janeiro (6,8\%) (Motta, 2012).

De acordo com pesquisa do Centro Regional de Estudos para o Desenvolvimento da Sociedade da Informação (CETIC) (Brasil, 2017a), com dados de 2014, mais da metade dos alunos de escolas em meios urbanos tinham acesso a um computador em casa $(57,2 \%)$, enquanto na área rural apenas $28,6 \%$. No ambiente escolar, segundo dados do Censo Escolar, realizada pelo Instituto Nacional de Estudos e Pesquisas Educacionais Anísio Teixeira (INEP) em 2019, a disponibilidade de internet oferecida aos alunos nas escolas de ensino fundamental era 32,1\%, enquanto para o ensino médio 62,3\% (Brasil, 2019).

O debate acerca do impacto das desigualdades no acesso à internet ganhou destaque no primeiro semestre de 2020, com a suspensão das aulas presenciais em muitos países, adotada como medida para contenção da propagação do vírus SARSCOV-2, declarada como pandemia pela Organização Mundial de Saúde em 11 de Março de 2020 (WHO, 2020). A suspensão 
prolongada das atividades presenciais de ensino, demandou uma adaptação abrupta das diferentes comunidades escolares, com uso intensivo de tecnologias remotas no processo de ensino aprendizagem.

No estado do Paraná, um decreto estadual no 4230, suspendeu as aulas das escolas estaduais por tempo indeterminado. Durante a pandemia do COVID-19, o governo do estado, disponibilizou para os estudantes da rede pública, aulas online por meio de um aplicativo, e em canais de TV vinculados a uma emissora de sinal aberto de TV. Estas alternativas irão ser mantidas enquanto perdurar a crise do coronavírus no país de acordo com o decreto estadual nº4230 (Paraná, 2020a).

Ainda que Curitiba, seja considerada uma das capitais brasileiras com melhores condições de vida (Paraná, 2020b), o município apresenta importantes desigualdades no acesso a diferentes estruturas, como por exemplo, ambiente construído para atividade física (Santos et al., 2019) parcialmente explicadas pela renda e distância do centro da cidade; e disponibilidade de espaços públicos para comercialização de frutas e hortaliças (Silva et al., 2020), em que as piores condições de acesso foram encontradas nas regiões periféricas, e com menor renda.

Apesar da disponibilidade de relatórios técnicos de instituições como CETIC e Instituto Brasileiro de Geografia e Estatística, bem como do INEP coletar anualmente informações acerca do acesso à internet no Censo Escolar (Brasil, 2019), o teste formal de hipóteses acerca da distribuição espacial de acesso à internet entre adolescentes não foi identificado em artigos científicos, bem como a existência de diferenças intramunicipais de acesso. Investigar estas dimensões do acesso, a partir da investigação de diferenças intramunicipais permite melhor conhecer a distribuição na cidade, e subsidiar ações de ampliação do acesso mais equânimes.

Desta forma, o objetivo do presente trabalho foi analisar as condições de acesso à internet por adolescentes da rede estadual de ensino de Curitiba, Paraná, e identificar desigualdades de acordo com características demográficas dos alunos e condições socioeconômicas do entorno escolar.

\section{Metodologia}

Para o presente artigo foram utilizados dados do projeto "Excesso de peso e características do ambiente escolar em estudantes de Curitiba, Paraná", com dados coletados entre março de 2016 e maio de 2017. Neste estudo quantitativo, observacional, de caráter transversal, analítico (Rothman et al., 2008; Köche, 2011; Medronho et al., 2009), foram avaliados alunos do $6^{\circ}$ ano do Ensino Fundamental ao $3^{\circ}$ ano do Ensino Médio de instituições públicas de ensino estadual de Curitiba, Paraná. Para a coleta de dados houve aplicação de questionário preenchido pelos estudantes e aferição de dados antropométricos.

Em 2016, a população de Curitiba foi estimada em 1.893.977 habitantes, sendo que destes, 221.952 eram estudantes do Ensino Fundamental e 78.815 do Ensino Médio (Brasil, 2016). Segundo o Censo Escolar de 2015 da Secretaria de Estado da Educação do Paraná (Paraná, 2015), em Curitiba havia 149 escolas públicas estaduais de Ensino Fundamental e 129 de Ensino Médio (Brasil, 2016). Foram consideradas na pesquisa as escolas estaduais de $6^{\circ}$ ano do Ensino Fundamental ao $3^{\circ}$ ano do Ensino Médio e excluídas as unidades de educação exclusiva especial ou indígena, totalizando 160 escolas estaduais elegíveis (Paraná, 2015). O número de alunos matriculados na rede pública estadual de ensino, do $6^{\circ}$ ano do Ensino Fundamental ao $3^{\circ}$ ano do Ensino Médio era de 143.701. Contudo, para a execução do projeto foi tomado como base a quantidade de estudantes destes anos, somente matriculados em turnos diurnos, totalizando 110.238 alunos.

O cálculo amostral foi realizado no programa OpenEpi, na Versão 3 - on line, de livre acesso, pela utilizando-se a fórmula: "n $=\left[\operatorname{EDFF}^{*} \mathrm{~Np}(1-\mathrm{p})\right] /\left[\mathrm{d} 2 / \mathrm{Z} 21-\alpha / 2 *(\mathrm{~N}-1)+\mathrm{p}^{*}(1-\mathrm{p})\right]$ ". Buscando maximizar o tamanho amostral foi utilizada prevalência desconhecida do desfecho de 50\%, com margem de erro de quatro pontos percentuais e nível de confiança de $95 \%$, resultando em uma amostra de 597 estudantes. Considerando o efeito de delineamento de 2, a amostra foi duplicada resultando em 1.194 estudantes. A fim de sanar resultados de eventuais perdas ou recusas, foi acrescentado $20 \%$ a este valor. Assim, o 
tamanho amostral final foi de 1.433 estudantes. Com esta estimativa amostral foi possível identificar razão de prevalência de 1,14, equivalente à prevalência de $50 \%$ entre expostos e $44 \%$ não-expostos, com poder de $80 \%$ e nível de confiança de $95 \%$.

Com o tamanho de amostra definido optou-se por realizar amostragem de aproximadamente mesmo tamanho em cada escola, que fosse viável para os pesquisadores, que consideraram $n=48$, adequado, resultando em aproximadamente 30 unidades escolares. As escolas foram listadas sequencialmente em ordem alfabética e sorteadas, em seguida foram sorteados os anos escolares a serem avaliados em cada escola. A distribuição contemplou todas as dez regionais administrativas do município com no mínimo duas e no máximo seis escolas. Em cada escola, nas turmas definidas para participar, o número de alunos da listagem foi identificado, e caso superior à amostra pretendida, houve sorteio para participação. Alunos ausentes na data de coleta de dados, e que não quiseram preencher o questionário foram considerados como perdas ou recusas, respectivamente.

Todos os participantes da coleta de dados foram treinados. O pré-teste do questionário foi realizado com adolescentes selecionados por conveniência. O estudo piloto foi realizado em escola não sorteada para a pesquisa, com aplicação do questionário e coleta de dados antropométricos de alunos de uma turma de 35 adolescentes de $1^{\circ}$ ano do Ensino Médio matutino, com idade entre 14 e 16 anos.

O questionário preenchido pelos alunos foi composto por questões de múltipla escolha e aplicado com a supervisão da pesquisadora principal durante o horário das aulas, em sala de aula. O instrumento, padronizado e estruturado, foi entregue em versão impressa aos alunos selecionados.

Inicialmente os alunos responderam às perguntas demográficas como: sexo (masculino, feminino), faixa etária (10-13 anos, 14-16 anos e 17 ou mais) e turno (matutino, vespertino e intermediário/integral). A seguir responderam sobre o tempo de deslocamento no trajeto casa/escola a pé ( 0 a $10 \mathrm{~min}, 11$ a $20 \mathrm{~min}, 21$ a $30 \mathrm{~min}, 31$ a $59 \mathrm{~min}$ e uma hora ou mais).

As condições de acesso à internet foram investigadas por meio de questionário, incluindo: 1) dispositivo mais frequente de uso (computador, celular, tablets, outro); 2) locais de acesso: casa, trabalho, escola, redes wi-fi, lan house, casa de amigos ou parentes, outros; 3) condições de uso: sozinho, dispositivo próprio; sozinho, uso compartilhado; acompanhado de irmãos ou amigos; acompanhado de pais e/ou responsáveis; e 4) frequência de uso da internet: diária, 5 x/sem, 3 x/sem, 1 x/sem, ou $1 \mathrm{x}$ por mês.

A renda do entorno escolar foi obtida por meio de dados secundários do Censo Demográfico (2010). Com auxílio da ferramenta de interseção do Qgis, os setores censitários contidos nos buffers de 500m do entorno escolar foram selecionados ( $\mathrm{n}$ = 375), e os dados de renda foram obtidos da base de dados do Instituto Brasileiro de Geografia e Estatística (Brasil, 2010). Para a definição da renda dos entornos escolares, foi calculada a média do rendimento nominal dos responsáveis por domicílio do conjunto de setores censitários que compunham cada área, provenientes do Censo Demográfico de 2010.

Modelos de regressão logística multinível em três níveis 1) adolescente, 2) turma; 3) escola permitiram estimar as razões de chance (OR) e intervalos de confiança de 95\% (IC95\%). OR não ajustadas foram estimadas para variáveis dos alunos, e das escolas para uso de computador e celular. Adicionalmente, as OR de uso diário, dispositivo próprio e uso acompanhado dos pais em relação à renda foram estimadas. As estimativas foram corrigidas para efeito do delineamento do estudo, e pesos amostrais. As análises foram realizadas no aplicativo Stata 15.

Foram elaborados mapas temáticos para identificar as relações entre renda e proporção de alunos com acesso à internet preferencial por computador, celular, acesso diário, condições de uso, ponderando as escolas pela distribuição da renda. Todos os procedimentos da estatística espacial foram desenvolvidos com auxílio do software QGIS 3.10 Coruña. A análise da correlação espacial de Moran foi efetuada por intermédio do software GeoDa. Na análise espacial de Moran foram executadas 99 permutações, aplicada a distância euclidiana e o método k-neighbors. Ao final dessa etapa foram geradas a associações espacial global (Índice de Moran) e local (LISA). 
Os indicadores globais como o Índice Global de Moran, fornecem um único valor como medida da associação espacial para todo o conjunto de dados, identificando a relação de interdependência entre as variáveis. Entretanto, para examinar os padrões de dados espaciais em uma escala de maior detalhe, quando a dependência espacial é mais acentuada, é recomendado o Indicador Local de Associação Espacial - LISA, que produz um valor específico para cada objeto, permitindo assim, a identificação de agrupamentos de objetos com valores de atributos semelhantes ou objetos anômalos, ou seja, a relação de vizinhança pela covariância (Luzardo et al., 2017; Nunes, 2013).

A pesquisa foi aprovada pelo Comitê de Ética em Pesquisa em Seres Humanos do Setor de Ciências da Saúde (CEP/SD) da UFPR (protocolo nº1426615/2016), conforme resolução 466/12 do Conselho Nacional de Saúde.

\section{Resultados}

Foram convidados a participar da pesquisa 1.623 alunos, dos quais 1.232 aceitaram (proporção de resposta 75,9\%); distribuídos homogeneamente em relação ao sexo (51,2\% masculino); 49,9\% estavam na faixa etária de 10 a 13 anos; e 57,0\% estudavam no turno matutino. O dispositivo empregado com maior frequência para acessar à internet foi o celular $(75,4 \%)$, seguido do computador $(27,3 \%)$ e dos tablets $(8,4 \%)$. A maioria dos adolescentes relatou acessar à internet em casa $(81,0 \%)$, ou por meio de redes wi-fi $(25,1 \%)$. Mais da metade $(53,1 \%)$ referiu acessar sozinho, em dispositivos próprios, enquanto 28,3\% relataram compartilhar com irmãos, e 10,7\% acompanhados dos pais e/ou responsáveis; 77,2\% referiram acesso diário à internet, enquanto $2,7 \%$ apenas uma vez ao mês (Tabela 1).

Tabela 1. Distribuição das características, e razão de chances (OR) de uso de computador ou celular para acessar internet e características demográficas e condições de acesso dos alunos, e do entorno escolar em escolas estaduais de Curitiba, PR, 2016-2017 ( $\mathrm{N}=1.233)$.

\begin{tabular}{|c|c|c|c|c|c|}
\hline Variáveis & & Computador* & & Celular** & \\
\hline & $\%(\mathrm{IC} 95 \%) *$ & OR $(95 \% \mathrm{CI})$ & $\begin{array}{c}\text { valor de } \\
p^{* * *}\end{array}$ & OR $(95 \% \mathrm{CI})$ & $\begin{array}{c}\text { valor de } \\
\mathrm{p}^{* * *}\end{array}$ \\
\hline Sexo & & & $<0,001$ & & $<0,001$ \\
\hline Masculino & $51,2(45,2 ; 57,2)$ & 1 & & 1 & \\
\hline Feminino & $48,8(42,8 ; 54,8)$ & $0,37(0,26 ; 0,51)$ & & $2,49(1,66 ; 3,73)$ & \\
\hline Faixa etária & & & 0,048 & & 0,002 \\
\hline 10-13 anos & $49,9(31,9 ; 67,9)$ & 1 & & 1 & \\
\hline 14-16 anos & $41,0(26,9 ; 56,9)$ & $0,90(0,64 ; 1,28)$ & & $1,44(1,02 ; 2,04)$ & \\
\hline 17 ou mais & $9,1(4,9 ; 16,2)$ & $0,55(0,32 ; 0,95)$ & & $2,65(1,30 ; 5,42)$ & \\
\hline Turno & & & 0,174 & & $<0,001$ \\
\hline Matutino & $57,0(35,4 ; 76,3)$ & 1 & & 1 & \\
\hline Vespertino & $34,5(16,9 ; 57,7)$ & $1,31(0,84 ; 2,04)$ & & $0,76(0,54 ; 1,05)$ & \\
\hline Intermediário/integral & $8,5(2,1 ; 28,9)$ & $1,50(0,78 ; 2,89)$ & & $0,38(0,23 ; 0,64)$ & \\
\hline Tempo deslocamento casa-escola & & & 0,337 & & 0,049 \\
\hline $0-10 \min$ & $39,4(30,8 ; 48,8)$ & 1 & & 1 & \\
\hline $11-20 \min$ & $24,2(20,8 ; 28,0)$ & $1,32(0,87 ; 2,02)$ & & $1,06(0,73 ; 1,54)$ & \\
\hline $21-30 \min$ & $15,2(12,3 ; 18,5)$ & $0,99(0,51 ; 1,9)$ & & $1,37(0,80 ; 2,35)$ & \\
\hline $31 \mathrm{~min}$ ou mais & $21,2(13 ; 32,6)$ & $0,72(0,43 ; 1,21)$ & & $1,65(0,98 ; 2,79)$ & \\
\hline \multicolumn{6}{|l|}{ Dispositivos acesso internet } \\
\hline Computador & $27,3(23 ; 32,1)$ & & & $0,03(0,01 ; 0,1)$ & $<0,001$ \\
\hline Celular & $75,4(71,3 ; 79,2)$ & $0,03(0,01 ; 0,09)$ & $<0,001$ & & \\
\hline Tablet & $8,4(5,5 ; 12,6)$ & $2,13(1,50 ; 3,02)$ & $<0,001$ & $0,32(0,19 ; 0,56)$ & $<0,001$ \\
\hline
\end{tabular}




\begin{tabular}{lllllr}
\hline Outro & $4,5(2,5 ; 8,0)$ & & & & \\
Locais de acesso internet & & & & & \\
Casa & $81(76,8 ; 84,6)$ & $1,96(1,29 ; 2,97)$ & 0,003 & $0,77(0,49 ; 1,23)$ & 0,267 \\
Trabalho & $4,3(2,6 ; 6,8)$ & $1,67(0,90 ; 3,07)$ & 0,099 & $0,91(0,46 ; 1,79)$ & 0,769 \\
Escola & $10,4(8,3 ; 13,0)$ & $1,39(0,83 ; 2,32)$ & 0,200 & $2,13(0,91 ; 4,95)$ & 0,078 \\
Redes wi-fi & $25,1(21,6 ; 28,9)$ & $1,08(0,79 ; 1,48)$ & 0,623 & $2,81(2,02 ; 3,92)$ & $<0,001$ \\
Lan house & $1,8(1,0 ; 3,0)$ & $1,52(0,59 ; 3,94)$ & 0,373 & $0,96(0,36 ; 2,53)$ & 0,928 \\
Casa amigos ou parentes & $19,8(16,7 ; 23,2)$ & $1,08(0,79 ; 1,48)$ & 0,623 & $1,53(0,93 ; 2,51)$ & 0,090 \\
Outros & $2,4(1,3 ; 4,3)$ & $0,79(0,39 ; 1,6)$ & 0,498 & $0,24(0,07 ; 0,81)$ & 0,022 \\
Condições uso & & & 0,986 & & 0,753 \\
Sozinho, dispositivo próprio & $53,1(45,8 ; 60,3)$ & & & & 1 \\
Sozinho, divide o uso & $28,3(24,4 ; 32,7)$ & $1,61(1,20 ; 2,15)$ & & $0,6(0,42 ; 0,86)$ & \\
Acompanhado irmãos ou amigos & $7,8(5,8 ; 10,5)$ & $1,65(0,84 ; 3,25)$ & & $0,66(0,35 ; 1,25)$ & \\
Acompanhado pais e/ou responsáveis & $10,7(7,6 ; 14,9)$ & $0,69(0,41 ; 1,14)$ & & $1,22(0,67 ; 2,23)$ & \\
Frequência de acesso & & & 0,95 & & \\
Diária & $77,2(69,9 ; 83,2)$ & & & & 1 \\
5 x/semana & $9,6(7,5 ; 12,1)$ & $0,89(0,5 ; 1,58)$ & & $0,68(0,35 ; 1,32)$ & \\
3 x/semana & $6,5(4,3 ; 9,7)$ & $1,27(0,66 ; 2,44)$ & & $0,43(0,27 ; 0,69)$ & \\
1 x/semana & $4,1(2,7 ; 6,1)$ & $1,49(0,68 ; 3,26)$ & & $0,21(0,11 ; 0,39)$ & \\
1 x/mês & $2,7(1,3 ; 5,3)$ & $0,4(0,09 ; 1,68)$ & & $0,57(0,26 ; 1,25)$ & \\
\hline
\end{tabular}

*Corrigido para efeito do delineamento do estudo e pesos amostrais;

IC95\% - intervalo de confiança de 95\%

** Renda dos domicílios particulares permanentes no raio de $500 \mathrm{~m}$ da escola.

***Regressão logística multinível. Nível 1= alunos, nível 2 = turma, nível 3= escola.

Fonte: Autores (2020).

As chances de usar o computador como principal meio de acesso à internet foi menor no sexo feminino, na faixa etária de 17 anos ou mais, e para os adolescentes que referiram usar o celular; no entanto, foi maior entre aqueles que referiram usar o tablet, ter acesso à internet no domicílio, e utilizar os dispositivos sem supervisão, mas compartilhar o uso do aparelho. Por outro lado, o uso do celular foi maior no sexo feminino, entre adolescentes com mais de 14 anos e que relataram acesso por meio de redes wi-fi; mas foi menor no turno intermediário/integral, entre aqueles que usavam celular ou tablet, que referiram uso compartilhado do aparelho, e acessar com menor frequência à internet (Tabela 2).

A chance de acessar internet por meio de computador foi menor para o tercil escolar de maior renda (OR 0,61 IC95\% 0,41; 0,91), assim como o uso acompanhado pelos pais e/ou responsáveis (OR 0,38 IC95\%0,16; 0,88); enquanto aumentaram com a renda as chances de acessar à internet: por meio de celular (OR 1,94 IC95\%1,25; 3,01), diariamente (OR 3,37; IC95\% 1,38; 8,20) e sozinho, por meio de dispositivos próprios (OR 1,81 IC95\% 1,00-3,27). (Tabela 2). 
Tabela 2. Razão de chances (OR) das condições de acesso à internet e características do entorno escolar em escolas estaduais de Curitiba, PR, 2016-2017.

\begin{tabular}{|c|c|c|}
\hline Variáveis & Computador & \\
\hline & OR $(95 \% \mathrm{CI}) *$ & valor de $\mathrm{p}^{*}$ \\
\hline Renda entorno escola & & 0,025 \\
\hline $1^{\circ}$ tercil $(246,2-474,7$ reais $)$ & 1 & \\
\hline $2^{\circ}$ tercil $(493,7-884,3$ reais $)$ & $1,26(0,82 ; 1,94)$ & \\
\hline \multirow[t]{2}{*}{$3^{\circ}$ tercil $(901,9-2.632,2$ reais $)$} & $0,61(0,41 ; 0,91)$ & \\
\hline & Celular & \\
\hline Renda entorno escola & & 0,005 \\
\hline $1^{\circ}$ tercil $(246,2-474,7$ reais $)$ & 1 & \\
\hline $2^{\circ}$ tercil $(493,7-884,3$ reais $)$ & $1,22(0,88 ; 1,69)$ & \\
\hline \multirow[t]{2}{*}{$3^{\circ}$ tercil $(901,9-2.632,2$ reais $)$} & $1,94(1,25 ; 3,01)$ & \\
\hline & Acesso Diário & \\
\hline Renda entorno escola & & 0,011 \\
\hline $1^{\circ}$ tercil $(246,2-474,7$ reais $)$ & 1 & \\
\hline $2^{\circ}$ tercil $(493,7-884,3$ reais $)$ & $0,94(0,46 ; 1,91)$ & \\
\hline \multirow[t]{2}{*}{$3^{\circ}$ tercil $(901,9-2.632,2$ reais $)$} & $3,37(1,38 ; 8,20)$ & \\
\hline & Uso sozinho, dispositivo próprio & \\
\hline Renda entorno escola & & 0,050 \\
\hline $1^{\circ}$ tercil $(246,2-474,7$ reais $)$ & 1 & \\
\hline $2^{\circ}$ tercil $(493,7-884,3$ reais $)$ & $1,04(0,62 ; 1,72)$ & \\
\hline \multirow[t]{2}{*}{$3^{\circ}$ tercil $(901,9-2.632,2$ reais $)$} & $1,81(1,00 ; 3,27)$ & \\
\hline & $\begin{array}{l}\text { Uso acompanhado pelos pais e/ou } \\
\text { responsáveis }\end{array}$ & \\
\hline Renda entorno escola & & 0,025 \\
\hline $1^{\circ}$ tercil $(246,2-474,7$ reais $)$ & 1 & \\
\hline $2^{\circ}$ tercil $(493,7-884,3$ reais $)$ & $0,81(0,39 ; 1,68)$ & \\
\hline $3^{\circ}$ tercil $(901,9-2.632,2$ reais $)$ & $0,38(0,16 ; 0,88)$ & \\
\hline
\end{tabular}

** Renda dos domicílios particulares permanentes no raio de $500 \mathrm{~m}$ da escola. Fonte: Autores (2020).

Os mapas que apresentam a distribuição das condições de acesso à internet nas escolas indicam concentração das escolas localizadas em territórios de maior renda nas áreas mais centrais do município, bem como maior proporção de acesso sozinho por meio de dispositivo próprio nas escolas de maior renda (Figura 1A e 1B). 
Figura 1. Proporção de adolescentes por escola estadual, dos turnos diurnos: A) por tipo de dispositivo preferencial de uso; B) por condições de uso de acordo com a renda no entorno da escola (n=30). Curitiba, Paraná, 2016-2017.

A)

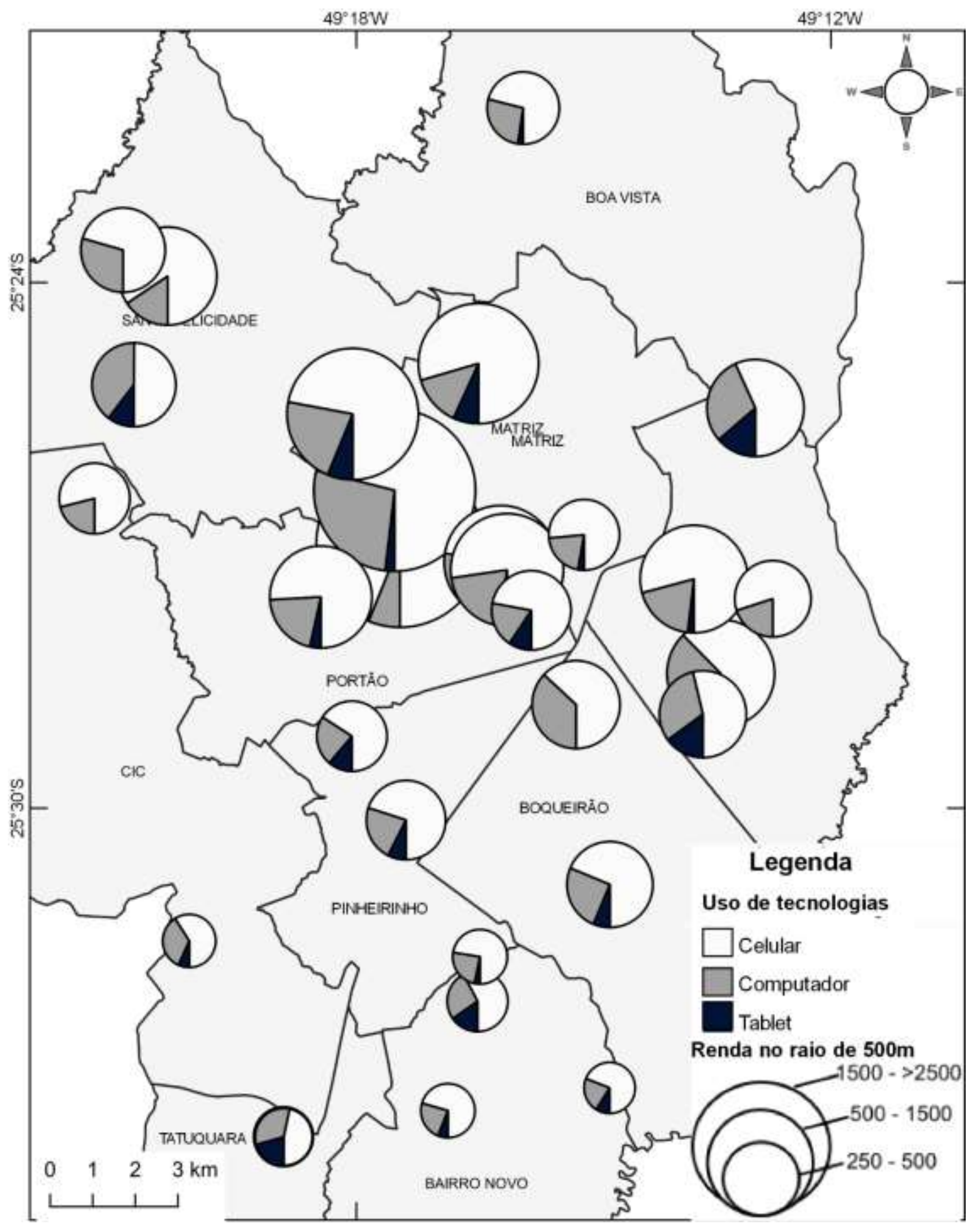


B)

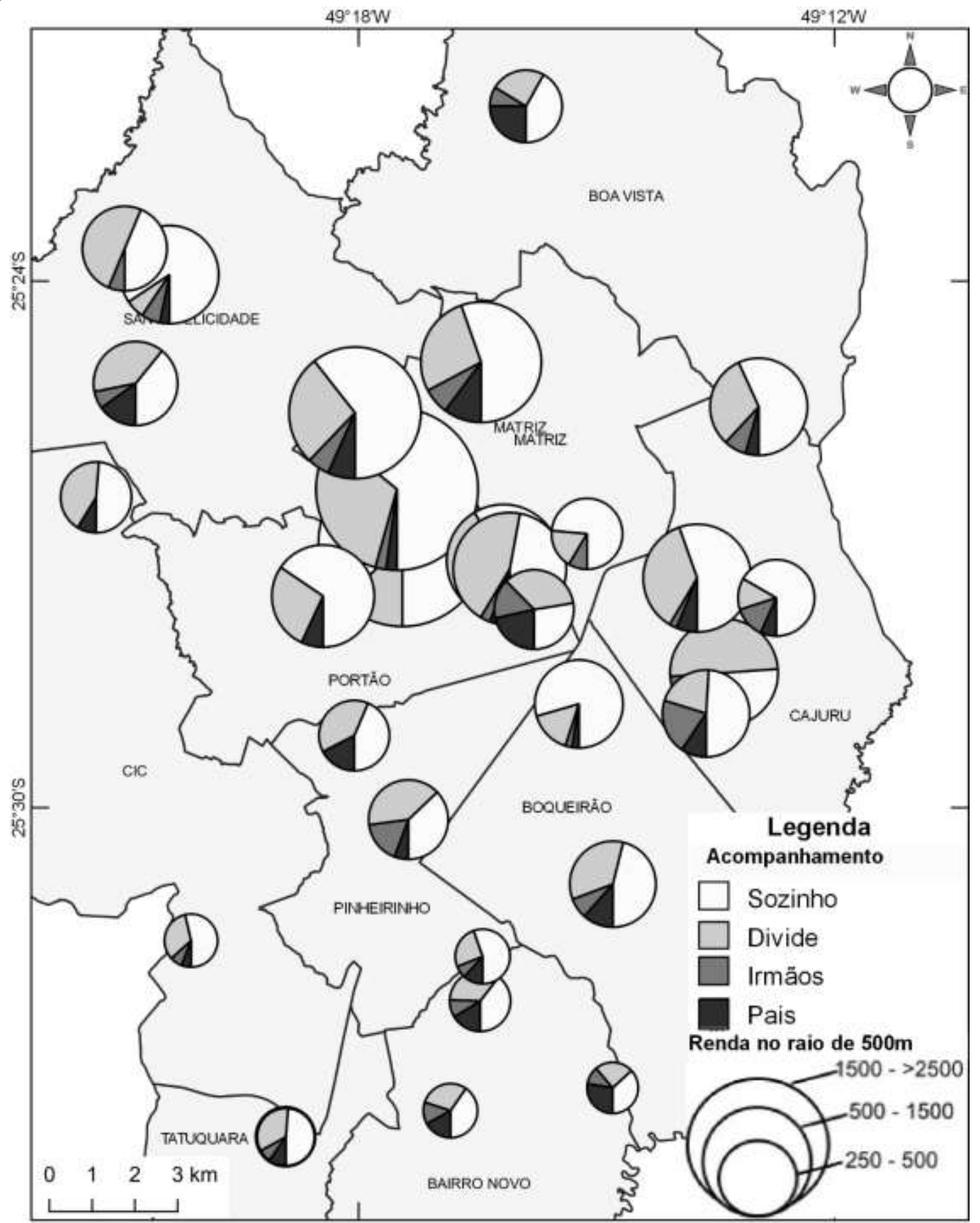

Fonte: Autores (2020).

O índice de Moran global, indicou correlação espacial inversa entre renda e uso do celular $(-0,26)$ e tablet $(-0,29)$, e positiva para computador $(0,31)$ e uso diário da internet $(0,30)$. O índice de Moran local identificou elevado número de escolas com clusters divergentes de acesso aos dispositivos e renda (alto-baixo, baixo-alto), especialmente para uso de computador, tablet, uso diário, sozinho e acompanhado pelos pais. Para uso de celular e computador cinco escolas apresentaram clusters do tipo baixo-baixo (Tabela 3 ). 
Tabela 3. Distribuição do índice de Moran global e local da renda do entorno das escolas com condições de acesso à internet em escolas estaduais ( $\mathrm{n}=30$ ) de Curitiba, PR. 2016-2017.

\begin{tabular}{|c|c|c|c|}
\hline Variáveis & $\begin{array}{c}\text { Índice de Moran } \\
\text { Global } \\
\end{array}$ & $\begin{array}{c}\text { LISA (moran) } \\
\text { Med (Min-Máx) }\end{array}$ & Classe \\
\hline \multirow[t]{4}{*}{ Celular } & $-0,26$ & $0,03(-1,23 ; 3,18)$ & $\mathrm{AA}=1$ \\
\hline & & & $\mathrm{AB}=3$ \\
\hline & & & $\mathrm{BA}=0$ \\
\hline & & & $\mathrm{BB}=5$ \\
\hline \multirow[t]{4}{*}{ Computador } & 0,31 & $-0,05(-2,22 ; 0,49)$ & $\mathrm{AA}=1$ \\
\hline & & & $\mathrm{AB}=3$ \\
\hline & & & $\mathrm{BA}=4$ \\
\hline & & & $\mathrm{BB}=5$ \\
\hline \multirow[t]{4}{*}{ Tablet } & $-0,29$ & $-0,13(-2,08 ;-0,33)$ & $\mathrm{AA}=2$ \\
\hline & & & $\mathrm{AB}=6$ \\
\hline & & & $\mathrm{BA}=4$ \\
\hline & & & $\mathrm{BB}=2$ \\
\hline \multirow[t]{4}{*}{ Uso Diário } & 0,30 & $0,00(-1,25 ; 2,24)$ & $\mathrm{AA}=1$ \\
\hline & & & $\mathrm{AB}=6$ \\
\hline & & & $\mathrm{BA}=4$ \\
\hline & & & $\mathrm{BB}=2$ \\
\hline \multirow[t]{4}{*}{ Uso sozinho } & 0,23 & $0,15(-0,78 ; 1,27)$ & $\mathrm{AA}=1$ \\
\hline & & & $\mathrm{AB}=6$ \\
\hline & & & $\mathrm{BA}=4$ \\
\hline & & & $\mathrm{BB}=2$ \\
\hline \multirow[t]{4}{*}{ Acompanhado pais } & $-0,20$ & $-0,04(-1,75 ; 1,33)$ & $\mathrm{AA}=1$ \\
\hline & & & $\mathrm{AB}=5$ \\
\hline & & & $\mathrm{BA}=4$ \\
\hline & & & $\mathrm{BB}=3$ \\
\hline
\end{tabular}

* Renda dos domicílios particulares permanentes no raio de $500 \mathrm{~m}$ da escola.

LISA = índice local de Moran; Med= Mediana; Min.= mínimo; Máx.= Máximo

$\mathrm{AA}$ - alto uso do dispositivo e alta renda; $\mathrm{AB}$ - alto uso do dispositivo e baixa renda; $\mathrm{BA}$ - baixo uso do dispositivo e alta renda; $\mathrm{BB}$ - baixo uso do dispositivo e baixa renda.

Fonte: Autores (2020).

\section{Discussão}

Os resultados do presente estudo indicaram que 1 em cada 5 adolescentes das escolas estaduais de Curitiba declarou não acessar internet no domicílio (19,0\%). Esta proporção é próxima àquela identificada pela PNAD em 2017 para o país, como um todo, indicando que 74,9\% dos domicílios do País possuíam acesso à internet, subindo para 79,1\% dos domicílios em 2018 (Brasil, 2017b).

A maioria dos adolescentes das escolas estaduais referiu acessar à internet por meio do uso de celulares, seguido de microcomputadores, e tablets. Metade referiu possuir dispositivo próprio e acessar sozinha à rede. O uso mais frequente do celular como meio de acessar à internet entre os adolescentes avaliados reflete o perfil de acesso da população brasileira, uma vez que em 2018 98,1\% referiu empregar o celular para este fim, 50,7\% o computador, 12,0\% o tablet, e 23,1\% a televisão (Brasil, 2010). Em um estudo realizado em Porto Alegre (2020) com adolescentes de 13 a 17 anos, 84,5\% relataram possuir computador em sua residência, e 95,1\% telefone celular de uso próprio para acesso à internet (Wendt et al., 2020).

Adolescentes do sexo feminino, e com maior idade, apresentaram maiores chances de referir o uso preferencial do celular, enquanto o uso do computador foi mais frequente no sexo masculino, e diminuiu com a idade. O aumento do uso do celular conforme o avanço da idade dos adolescentes também foi encontrado na PNAD (2018) (Brasil, 2017b), sendo que 43,5\% dos adolescentes de 10 a 13 anos tinham telefone móvel celular para uso pessoal, enquanto entre aqueles de 14 a 17 anos 73,6\% (Brasil, 2018). Padrões distintos de uso de internet por gênero foram identificados em estudos prévios (Brasil, 2018; Wendt et al., 2020). As diferenças observadas no presente estudo, como maior proporção de uso do computador no sexo 
masculino, e do celular no sexo feminino, são compatíveis com as finalidades de uso da internet entre os gêneros. No Brasil, em todas as práticas o maior consumo de internet pelo público do sexo masculino se destaca, principalmente para as ações de jogar online e baixar conteúdo de jogos e filmes (Silva et al., 2019).

A redução do custo relativo de smartphones, tem favorecido o acesso à internet por meio do uso do dispositivo, como um recurso de comunicação e obtenção de informações, integrando o cotidiano de parcela expressiva e crescente de pessoas (Brasil, 2018). Apesar disto, no Brasil, o uso do celular ocorre principalmente por meio da modalidade pré-paga, com limites baixos de uso de dados, os quais impõem barreiras à qualidade e ao tempo de conexão (Barbosa et al., 2010).

Desta forma, o uso do celular para acessar à internet, lança preocupações, especialmente na ausência de computadores disponíveis em casa para acompanhar as atividades escolares no domicílio, em relação aos impactos negativos associados ao seu uso no desempenho acadêmico identificado em estudantes de diferentes países (Felisoni \& Godoi, 2018; Lepp et al., 2015). Partes dos resultados educacionais negativos associados ao uso de telefones celulares estão relacionados ao estímulo à interação com outras pessoas, e ao comportamento multitarefa, os quais limitam a concentração para as atividades (Han \& Yi, 2019).

Entre os alunos, o acesso preferencial por meio de computador foi menor entre aqueles em escolas localizadas no maior tercil de renda, enquanto na análise espacial, houve associação positiva entre aumento da renda e proporção de uso do computador. Análises a partir dos dados da PNAD indicam o computador como meio de acesso associado à maior renda (Brasil, 2019; Brasil, 2018; Salata et al., 2013). A maior probabilidade de possuir acesso à microcomputador no núcleo das cidades, e/ou regiões de maior renda tem sido identificada a partir das análises de dados da PNAD. A localização do domicílio em área de favela reduziu em 37\% a probabilidade de ter computador; por outro lado, a localização do domicílio no núcleo das cidades, e não nas periferias aumentou em $24 \%$ essa chance (Salata et al., 2013).

A partir dos dados da pesquisa TIC Domicílios do CETIC, Mendonça \& Silva identificaram que quanto maior é o nível de escolaridade ou renda, maior é o efeito sobre a probabilidade de o domicílio conectar-se à internet (Mendonça \& Silva, 2019). Os alunos das escolas localizadas em regiões mais pobres, com maior frequência relataram não possuir dispositivos próprios para acessar à internet, ao invés disto, o uso se dava por meio de dispositivos de forma compartilhada e/ou supervisionada por irmãos, pais e amigos. No presente estudo, o acesso mais usual por meio de celular, com frequência diária, e com uso de dispositivo próprio foi mais comum nas escolas localizadas em regiões de maior renda. Desta forma, é possível que o uso preferencial do celular entre os adolescentes em escolas de maior renda para acessar à internet, reflita melhores condições financeiras, que possibilitem a conveniência e a privacidade do acesso.

O relatório da Organização para Cooperação e Desenvolvimento Econômico (OECD), utilizando dados de mais de 40 países, confirmou essa diferença no uso da internet entre adolescentes ricos e pobres, e que esta diferença reflete no desempenho acadêmico - a diferença do uso não está na possibilidade de acesso, e sim no conhecimento das potencialidades de pesquisa e acesso às informações (Peña-López et al, 2016).

Além das desigualdades de acesso nas condições de uso de acordo com a renda - possibilidade de acessar internet por celular, dispositivo próprio e acesso diário identificadas no presente estudo - é importante considerar outros aspectos não investigados, como o tipo de conexão banda larga, ou pacote de dados, e as habilidades para aproveitar as diversas oportunidades na conexão, as quais dependem de múltiplos recursos materiais e imateriais, e podem potencializar, ou reduzir as oportunidades do acesso à internet sobre as trajetórias de vida dos indivíduos (Salata et al., 2013). Indivíduos com maior renda e escolaridade possuem maior probabilidade de utilizar as tecnologias com maior facilidade e aproveitamento das informações (Salata et al., 2013; Hargittai, 2008). De acordo com um estudo longitudinal realizado na Suíça (2017), as crianças demonstraram usar a internet como forma de entretenimento e comunicação; e quanto menor a renda da família, esse uso da internet em entretenimento era mais frequente, sugerindo a necessidade de uma educação digital para toda a família, como 
forma de melhorar o aproveitamento do uso, contribuindo para a educação e melhor aproveitamento escolar das crianças e adolescentes (Camerini et al., 2018)

Salata et al. (2013) criaram três tipologias de acesso à internet, de acordo com as finalidades de usos mais comuns dos usuários, e identificaram que os perfis considerados intermediários, os quais se diferenciam do básico, pela realização de atividades como comunicação, educação, leitura (jornais e revistas) e lazer , e o perfil considerado avançado, que compreende atividades como compras online, transações bancárias e interações com órgãos governamentais, foram mais frequentes nas classes de maior renda e escolaridade. Ainda, as limitações dos dispositivos, da qualidade da conexão, e das possibilidades de uso entre aqueles com pior renda, podem estar relacionadas ao menor bem estar psicológico (George et al., 2020).

Adicionalmente, na situação de pandemia e uso intensivo de tecnologias de informação no processo ensinoaprendizagem, as desigualdades de acesso identificadas, incluindo o compartilhamento dos dispositivos no domicílio outros moradores, necessitando do computador para atividades laborais, bem como mais de um aluno no mesmo domicílio, podem agravar as iniquidades sociais.

Ainda, a sobrecarga do sexo feminino com afazeres domésticos (Brasil, 2010) tende a ser exacerbada neste período. A interrupção das aulas que atinge crianças, outros adolescentes residentes no mesmo domicílio, ou do contexto familiar mais amplo, bem como a flexibilização das condições de isolamento social com retorno de muitas mulheres adultas nos domicílios para seus postos de trabalho, aumentam as probabilidades de que as adolescentes do sexo feminino assumam de forma intensiva, uma parcela ainda maior de afazeres domésticos e cuidados com outros moradores, e encontrem obstáculos para a dedicação às atividades escolares remotas realizadas de forma online no período de suspensão das atividades didáticas presenciais.

Desta forma, as desigualdades econômicas que dificultam o acesso à internet entre os adolescentes podem privá-los de desenvolver habilidades, assim como estudar e buscar melhores informações e desempenho escolar. Essas desigualdades podem estar relacionadas não somente à renda e ao nível de educação, mas também ao gênero, raça/cor e local de moradia destes adolescentes (Robinson et al., 2015 ;Souza et al., 2021).

Apesar de os estudos indicarem o efeito mais pronunciado das variáveis do nível individual nas desigualdades sociais, o efeito contextual, relacionado à relação entre a localização dos grupos sociais no espaço, e das desvantagens criadas pela formatação de contextos sociais homogêneos nos quais os comportamentos são encorajados ou coibidos (Salata et al., 2013), os achados do presente estudo reforçam as desvantagens do acesso entre os adolescentes em escolas localizadas em regiões com pior renda, com destaque para as dificuldades de acesso via celulares à internet, uso compartilhado dos dispositivos, e menor frequência de acesso.

No questionário do presente estudo, os adolescentes foram estimulados, a escolher apenas a opção de uso mais frequente de internet, e não a informar sobre a disponibilidade de cada um dos dispositivos para acessar à rede, desta forma, é possível que nas regiões de maior renda, os alunos que referiram acessar à internet preferencialmente pelo celular, também possuam microcomputadores em seus domicílios. A disponibilidade dos dados de renda dos núcleos familiares dos alunos, poderia auxiliar na exploração destas divergências entre análises individuais e espaciais. Apesar da ausência de informações sobre renda dos domicílios, é importante destacar que se trata de alunos de escolas públicas, que provavelmente não reflita a distribuição da renda na população, especialmente, entre os maiores patamares de renda, o que pode influenciar os resultados.

Ainda que no presente estudo não tenha sido possível analisar o acesso a partir das variáveis socioeconômicas do nível individual, em função da reduzida proporção de resposta dos questionários encaminhados aos pais e responsáveis pelos adolescentes, as informações socioeconômicas das escolas, e análises espaciais, permitiram identificar as iniquidades socioeconômicas no acesso à internet, com padrões distintos em relação à análise do nível do aluno, e espaciais. A maioria dos adolescentes relatou residir nas proximidades das escolas o que indica a possibilidade de que residam em domicílios que 
compartilhem as condições socioeconômicas do entorno escolar, para além disso, as escolas são consideradas polos geradores de tráfego (Neves, 2015), e os espaços em seu entorno são utilizados pelos alunos em seus deslocamentos, e atividades cotidianas.

Cumpre destacar, que as informações empregadas para as análises do presente estudo são provenientes de 2016, e 2017 e que podem ter ocorrido mudanças nas proporções de acesso à internet, e outras condições de uso entre os adolescentes das escolas públicas. O estudo também está sujeito às limitações associadas ao preenchimento do questionário pelos próprios adolescentes. Contudo, a equipe de pesquisadores de campo esteve presente em sala de aula, e disponível para esclarecer as dúvidas dos alunos.

Adicionalmente, os padrões de uso de adolescentes, as mudanças temporais no incentivo ao uso de smartphones, bem como a existência de clusters discordantes de proporção de acesso e renda nas análises espaciais podem auxiliar na explicação dos padrões distintos encontrados a partir dos dados individuais e espaciais. Na área da saúde, a teoria da equidade inversa, propõem que quando as intervenções são implantadas elas alcançam inicialmente, os grupos com melhores condições socioeconômicas, e só posteriormente se expandem aos mais pobres (Victora et al., 2000). Assim, seria esperado que os domicílios com maior renda, fossem inicialmente beneficiados pelas políticas de ampliação do acesso aos smartphones, e banda larga, por exemplo. Desta forma, reduzir as iniquidades sociais requer investimentos para tornar as melhores condições de acesso mais amplamente acessíveis aos mais pobres.

Silva et al. (2019), destacam a importância de relativizar os discursos hegemônicos que pretendem atribuir caráter democratizante das tecnologias da informação, “(...) pois a internet produz e reproduz hierarquias, exclusões e desigualdades sociais". Ainda, os autores destacam a necessidade de compreensão sobre os determinantes das práticas e características culturais que estão relacionadas aos investimentos individuais no uso de tecnologias de informação e comunicação (Silva et al., 2019).

\section{Considerações Finais}

Os achados do presente estudo demonstraram desigualdades nas condições de acesso à internet de acordo com características dos adolescentes - como sexo, e faixa etária - e do entorno das escolas, com diferenças socioeconômicas no tipo de dispositivo, frequência de uso e disponibilidade de dispositivo próprio.

Estudos futuros, podem identificar as mudanças nas condições de acesso à internet entre os adolescentes de escolas estaduais ao longo do tempo, e investigar se características socioeconômicas individuais moderam as condições de acesso do nível da escola, domicílio ou outra unidade geográfica de interesse, bem como analisar os clusters que exibem padrões divergentes das condições de acesso e renda.

Por fim, as desigualdades nas condições de acesso observadas entre os adolescentes, devem ser consideradas pelos responsáveis pelas políticas educacionais, especialmente aos adolescentes das escolas localizadas em regiões mais pobres, para reduzir as desigualdades no acesso aos recursos tecnológicos, e nas habilidades para seu aproveitamento para não agravar ainda mais as profundas iniquidades educacionais existentes no país.

\section{Referências}

Barbosa, A. F., Cappi, J., Tavares, R., Gorroux, C., Oyadomari, W., \& Jereissati, T. (2010). Panorama Setorial da Internet, Redes sociais: revolução cultural na internet. CGI. br: Comitê Gestor da Internet no Brasil, 15.

Brasil. (2010). Instituto Brasileiro de Geografia e Estatística (IBGE). Censo Demográfico 2010. Famílias e domicílios. https://censo2010.ibge.gov.br Brasil. (2016). Instituto Brasileiro de Geografia e Estatística (IBGE). Pesquisa Nacional de Saúde dos Escolares (PeNSE).

Brasil. (2017a). Centro Regional para o Desenvolvimento da Sociedade da Informação (CETIC). São Paulo. https://cetic.br/pt/pesquisa/dom 
icilios/indicadores/

Brasil. (2017b). Instituto Brasileiro de Geografia e Estatística (IBGE). PNADC: Acesso à internet e à televisão e posse de telefone móvel celular para uso pessoal.

Brasil. (2018). Instituto Brasileiro de Geografia e Estatística (IBGE). Pesquisa Nacional por Amostra de Domicílios contínua. https://biblioteca.ibge.gov.br/visualizacao/livros/liv101650_informativo.pdf \%3E.

Brasil. (2019). Instituto Nacional de Estudos e Pesquisas Educacionais Anísio Teixeira (INEP). Censo Escolar. Brasilia. http://portal.inep.gov.br/censoescolar

Camerini, A.-L., Schulz, P. J., \& Jeannet, A.-M. (2018). The social inequalities of Internet access, its use, and the impact on children's academic performance: Evidence from a longitudinal study in Switzerland. New Media $\backslash \&$ Society, 20(7), 2489-2508.

da Silva, F. A. B., Ziviani, P., \& Ghezzi, D. R. (2019). As tecnologias digitais e seus usos.

de Souza, G. H. S., Jardim, W. S., Marques, Y. B., Junior, G. L., dos Santos, A. P. S., \& de Paula Liberato, L. (2021). Educação Remota Emergencial (ERE): Um estudo empírico sobre Capacidades Educacionais e Expectativas Docentes durante a Pandemia da COVID-19. Research, Society and Development, 10(1), e37510111904--e37510111904.

Felisoni, D. D., \& Godoi, A. S. (2018). Cell phone usage and academic performance: An experiment. Computers $\backslash \&$ Education, 20(7), 2489-2508. https://doi.org/10.1016/j.compedu.2017.10.006

George, M. J., Jensen, M. R., Russell, M. A., Gassman-Pines, A., Copeland, W. E., Hoyle, R. H., \& Odgers, C. L. (2020). Young adolescents' digital technology use, perceived impairments, and well-being in a representative sample. The Journal of pediatrics, 219, $180-187$.

Han, S., \& Yi, Y. J. (2019). How does the smartphone usage of college students affect academic performance? Journal of Computer Assisted Learning, 35(1), $13-22$.

Hargittai, E. (2008). The digital reproduction of inequality. In Social stratification (p. 936-944). Westview Press.

Klein, V., dos Santos, C. V., \& Souza, D. M. (2019). Recursos tecnológicos: avaliando o acesso de estudantes do ensino fundamental. Redin-Revista Educacional Interdisciplinar, $8(1)$.

Köche, J. C. (2011). Fundamentos de metodologia cientifica: teoria da ciência e iniciação à pesquisa. Vozes. http://www.brunovivas.com/wpcontent/uploads/sites/10/2018/07/Köche-José-Carlos0D0AFundamentos-de-metodologia-científica-_-teoria-da0D0Aciência-e-iniciação-à-pesquisa.pdf

Lepp, A., Barkley, J. E., \& Karpinski, A. C. (2015). The relationship between cell phone use and academic performance in a sample of US college students. Sage Open, 5(1), 2158244015573169.

Luzardo, A. J. R., Castañeda Filho, R. M., \& Rubim, I. B. (2017). Análise espacial exploratória com o emprego do Índice de Moran. GEOgraphia, 19(40), $161-179$.

Medronho, R. de A., Bloch, K. V., Raggio, L. R., \& Werneck, G. L. (2009). Epidemiologia (2 ed.). Atheneu.

Mendonça, M. J., \& Silva, J. J. da. (2019). Estimação da demanda de internet no Brasil.

Motta, M. P. da. (2012). Os acessos fixos à internet no Brasil e suas tecnologias. Confins. Revue franco-brésilienne de géographie/Revista franco-brasilera de geografia, 15. https://journals.openedition.org/confins/7592

Neves, F. H. (2015). Planejamento de equipamentos urbanos comunitários de educação: algumas reflexões. Cadernos Metrópole, $17(34)$, 503-516.

Nunes, F. G. (2013). Análise exploratória espacial de indicadores de desenvolvimento socioambiental das regiões de planejamento do norte e nordeste goiano.

Paraná. (2015). Secretaria de Estado da Educação do Paraná(SEED-PR). Superintendência de Desenvolvimento Educacional. Diretoria de Políticas e Tecnologias Educacionais. Consulta Escolas - 2014.

Paraná. (2020a). Decreto $n^{o} 4230$, de 16 de março de 2020. Dispõe sobre as medidas para enfrentamento da emergência de saúde pública de importância internacional decorrente do Coronavírus - COVID-19. Diário Oficial do Estado do Paraná.

Paraná. (2020b). Instituto Paranaense de Desenvolvimento Econômico e Social (IPARDES). Cadernos Municipais. http://www.ipardes.pr.gov.br/Pagina/Cadernos-municipais

Peña-López, I., \& others. (2016). Are there differences in how advantaged and disadvantaged students use the Internet? https://ictlogy.net/bibliography/reports/projects.php?idp=3245

Robinson, L., Cotten, S. R., Ono, H., Quan-Haase, A., Mesch, G., Chen, W., Schulz, J., Hale, T. M., \& Stern, M. J. (2015). Digital inequalities and why they matter. Information, communication $\backslash \&$ society, 18(5), 569-582.

Rothman, K. J., Greenland, S., \& Lash, T. L. (2008). Modern epidemiology. Lippincott Williams \& Wilkins.

Salata, A., Costa, L., Ribeiro, M. G., \& et al. (2013). Desigualdades digitais: acesso e uso da internet, posição socioeconómica e segmentação espacial nas metrópoles brasileiras. Análise social, 207, 288-320.

Santos, D. S. dos, Hino, A. A. F., \& Höfelmann, D. A. (2019). Iniquities in the built environment related to physical activity in public school neighborhoods in Curitiba, Paraná State, Brazil. Cadernos de saude publica, 35, e00110218. 
Research, Society and Development, v. 10, n. 2, e34410212703, 2021

(CC BY 4.0) | ISSN 2525-3409 | DOI: http://dx.doi.org/10.33448/rsd-v10i2.12703

Silva, A. D. da C. e, Silva, A. R. da, \& Höfelmann, D. A. (2020). Distribuição Espacial dos Equipamentos Públicos para Comercialização de Frutas, Legumes e Verduras em Curitiba, Paraná. Cien Saude Colet. http://www.cienciaesaudecoletiva.com.br/artigos/distribuicao-espacial-dos-equipamentospublicos-para-comercializacao-de-frutas-legumes-e-verduras-em-curitiba-parana/17591

Souza, L. G. S., \& Dumont, L. M. M. (2018). Exclusão e inclusão digitais em bibliotecas públicas municipais da Região Metropolitana de Belo Horizonte: análise do serviço de acesso à internet disponibilizado pelas instituições para o exercício da cidadania. Encontros Bibli: revista eletrônica de biblioteconomia e ciência da informação, 23(52), 48-60.

Victora, C. G., Vaughan, J. P., Barros, F. C., Silva, A. C., \& Tomasi, E. (2000). Explaining trends in inequities: evidence from Brazilian child health studies. The Lancet, 356(9235), 1093-1098.

Wendt, G. W., Appel, M., \& Koller, S. (2020). Tecnologias da informação e comunicação em adolescentes, práticas parentais e percepção de clima escolar: Uma abordagem multinível. Interação em Psicologia, 24(1).

World Health Organization. (2020). Statement on the second meeting of the International Health Regulations (2005) Emergency Committee regarding the outbreak of novel coronavirus (2019-nCoV). Statement on the second meeting of the International Health Regulations. https://www.who.int/newsroom/detail/30-01-2020-statement-on-the-second-meeting-of-the-international-health-regulations-(2005)-emergency-committee-regarding-the-outbreak-ofnovel-coronavirus-(2019-ncov) 